\title{
CÔNG CỤ CHUYỂN ĐỔI TỌA ĐỘ ONLINE PHỤC VỤ CHUYỂN ĐỔI TỌA ĐỘ QUA LẠI GIŨ̉A VN2000 VÀ WGS84
}

\author{
PGS. TS. TRẦN TRỌNG ĐỨC \\ Trường Đại học Bách Khoa - ĐHQG-HCM
}

\section{Tóm tắt:}

Theo quyết định số 83/2000/QĐ-TTg, tọa độ bản đồ được lưu trữ và thể hiện theo hệ quy chiếu và Hệ tọa độ quốc gia VN-2000. Thế nhưng trong thực tế ứng dụng, chẳng hạn trong lĩnh vực GPS, tọa độ được ghi nhận có thể là tọa độ trong hệ tọa độ quốc tế WGS84 trước khi được chuyển đổi về hệ tọa độ VN-2000. Việc chuyển đổi tọa độ qua lại có thể được thực hiện bởi một số phần mềm thương mại chuyên dụng. Tuy nhiên, thay vì phải trang bị phần mềm chuyên dụng, bài báo này đề xuất xây dựng một hệ thống chuyển đổi tọa độ online để chuyển đổi tọa độ thông qua internet. Hệ thống - được xây dụng theo mô hình client/server - cho phép người sử dụng chỉ với một trình duyệt web, như firefox, có thể tính chuyển tọa độ giữa hai hệ thống mà không cần đến một phần mềm chuyên dụng. Một thực nghiệm đã được tiến hành sử dụng hệ thống vừa xây dựng để chuyển đổi tọa độ của 100 điểm ngẫu nhiên trong phạm vi Việt Nam. Kết quả chuyển đổi tọa độ nhận được là phù hợp với kết quả nhận được khi sử dụng thư viện mã nguồn mở nổi tiếng Proj4js, qua đó khẳng định tính đúng đắn của hệ thống được đề xuất.

\section{1. Đặt vấn đề}

Các đối tượng trên Trái đất tồn tại và phân bố thực tế trên bề mặt cong và khi đề cập đến vị trí của chúng thì thường là những vị trí này được ghi nhận trong hệ tọa độ địa tâm 3 chiều hay hệ tọa độ trắc địa (đôi khi còn gọi là hệ tọa độ địa lý). Tuy nhiên, để tiện tham chiếu đến thì trong thực tế vị trí các đối tượng này lại được thể hiện trên bề mặt phẳng. Ở Việt Nam, từ năm 1972 hệ quy chiếu và hệ toạ độ quốc gia HN72 với mặt tham chiếu là ellipsoid Krasovky đã được sử dụng và kể từ năm 2000 theo Quyết định số 83/2000/QĐTTg ngày 12/07/2000 của Thủ tướng Chính phủ [6], tọa độ bản đồ được lưu trữ và thể hiện theo Hệ quy chiếu và Hệ tọa độ quốc gia VN-2000 với mặt tham chiếu là ellipsoid quốc tế WGS84. Bên cạnh việc ghi nhận và lưu trữ tọa độ theo hệ VN-2000, các thiết bị đo đạc theo công nghệ định vị GPS thu thập thông tin vị trí của các đối tượng theo hệ tọa độ quốc tế WGS84 và sau đó chuyển đổi tọa độ từ hệ này về hệ tọa độ địa phương, trong trường hợp của Việt Nam là hệ tọa độ VN-2000, để sử dụng. Nếu các đối tượng được tham chiếu và thể hiện trong một hệ quy chiếu duy nhất thì không gặp vấn đề, tuy nhiên khi các đối tượng được tham chiếu và thể hiện trong các hệ thống quy chiếu khác nhau được đặt cùng nhau sẽ phát sinh các vấn đề về khác biệt vị trí và khi đó đòi hỏi phải thực hiện các bài toán chuyển đổi tọa độ để đưa chúng về một hệ thống quy chiếu tọa độ chung. Do vậy, đối với trường hợp của Việt Nam, bài toán chuyển đổi tọa độ thường cần thực hiện là bài toán chuyển đổi tọa độ qua lại giữa hệ tọa độ quốc tế WGS84 và hệ VN-2000. Tuy nhiên, thay vì phải trang bị phần mềm chuyên dụng, bài báo này đề xuất một hệ thống chuyển đổi tọa độ online khai thác khả năng trao đổi thông tin tự do qua internet. Hệ thống chuyển đổi tọa

Ngày nhận bài: 28/3/2016

Ngày chấp nhận đăng: 06/5/2016 
độ online này sẽ hoạt động theo mô hình client-server giống như hoạt động của một website thông thường, hình 1 . Ở phía client, chỉ là một trình duyệt web như Internet Explorer, FireFox... để mở website hiển thị form tính chuyển tọa độ theo địa chỉ URL định sẵn. Ứng dụng phía Client được viết bằng javascript kết hợp sử dụng các công nghệ chuẩn của W3C (World Wide Web Consortium) nhằm tăng tính linh động cho web cũng như tăng tính tương tác với người duyệt web. Ở phía server là một Webserver, tiếp nhận và đáp lại những yêu cầu từ những trình duyệt web thông thường thông qua nghi thức truyền dữ liệu trên mạng HTTP. Web Server tiếp nhận các yêu cầu chuyển đổi tọa độ từ Client, xử lý yêu cầu và trả kết quả chuyển đổi tọa độ theo yêu cầu của Client. Web server sẽ trả thông tin về cho người duyệt web theo khuôn mẫu định sã̃n.

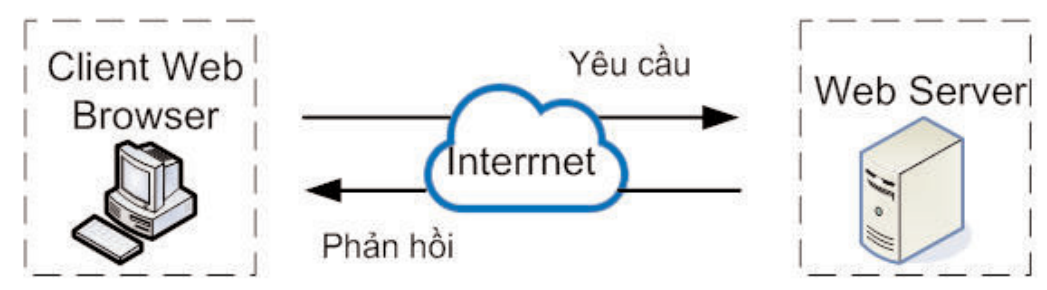

Hình 1: Kiến trúc của hệ thống chuyển đổi tọa độ online

Phần tiếp theo của bài báo sẽ trình bày cách thức tính chuyển tọa độ qua lại giữa hai hệ thống tọa độ và tiến hành xây dựng một công cụ chuyển đổi tọa độ online sử dụng ngôn ngữ javascript phía client và python phía server.

\section{Cơ sở lý thuyết trong chuyển đổi tọa độ giữa các hệ tọa độ}

Mọi đối tượng khi được thể hiện trên bản đồ, tọa độ đều gắn liền với một hệ tọa độ xác định. Về cơ bản, có 3 loại hệ thống tọa độ: Hệ tọa độ địa tâm, hệ tọa độ trắc địa và hệ tọa độ chiếu [1]. Dữ liệu được thành lập bởi nhiều tổ chức khác nhau, phục vụ cho nhiều mục đích khác nhau nên dữ liệu có thể tồn tại một cách đa dạng trong nhiều hệ thống tọa độ. Khi cần thiết có thể chuyển đổi dữ liệu từ một hệ thống tọa độ này sang một hệ thống tọa độ khác. theo Knippers, R.A and Hendrikse J[4], các phương pháp chuyển đổi tọa độ qua lại giữa các hệ thống có thể được tổng kết như hình 2

\section{Chuyển đổi tọa độ trắc địa $\mathrm{B}, \mathrm{L}$ ở WGS84 sang tọa độ phẳng $\mathrm{x}, \mathrm{y}$ ở VN-2000}

Bài toán chuyển đổi tọa độ này đòi hỏi phải kết hợp chuyển đổi tọa độ ở các bề mặt tham chiếu khác nhau - mặt tham chiếu A là WGS84 và mặt tham chiếu B là VN-2000 và chuyển đổi tọa độ trắc địa ở một mặt tham chiếu đến tọa độ phẳng theo 1 mặt tham chiếu khác. Dựa vào cơ sở lý thuyết trong chuyển đổi tọa độ tổng kết ở hình 2 , thì một trong những phương án có thể được áp dụng là quy trình tính chuyển 4 bước như trình bày dưới đây:

Bước 1: tính chuyển từ tọa độ trắc địa $\mathrm{B}, \mathrm{L}$ ở WGS84 sang tọa độ địa tâm XYZ ở WGS84, sử dụng công thức tính chuyển theo [3]:

$$
\begin{aligned}
& X=(N+H) \cos B \cos L ; \\
& Y=(N+H) \cos B \sin L ; \\
& Z=\left\{N\left(1-e^{2}\right)+H\right\} \sin B,
\end{aligned}
$$




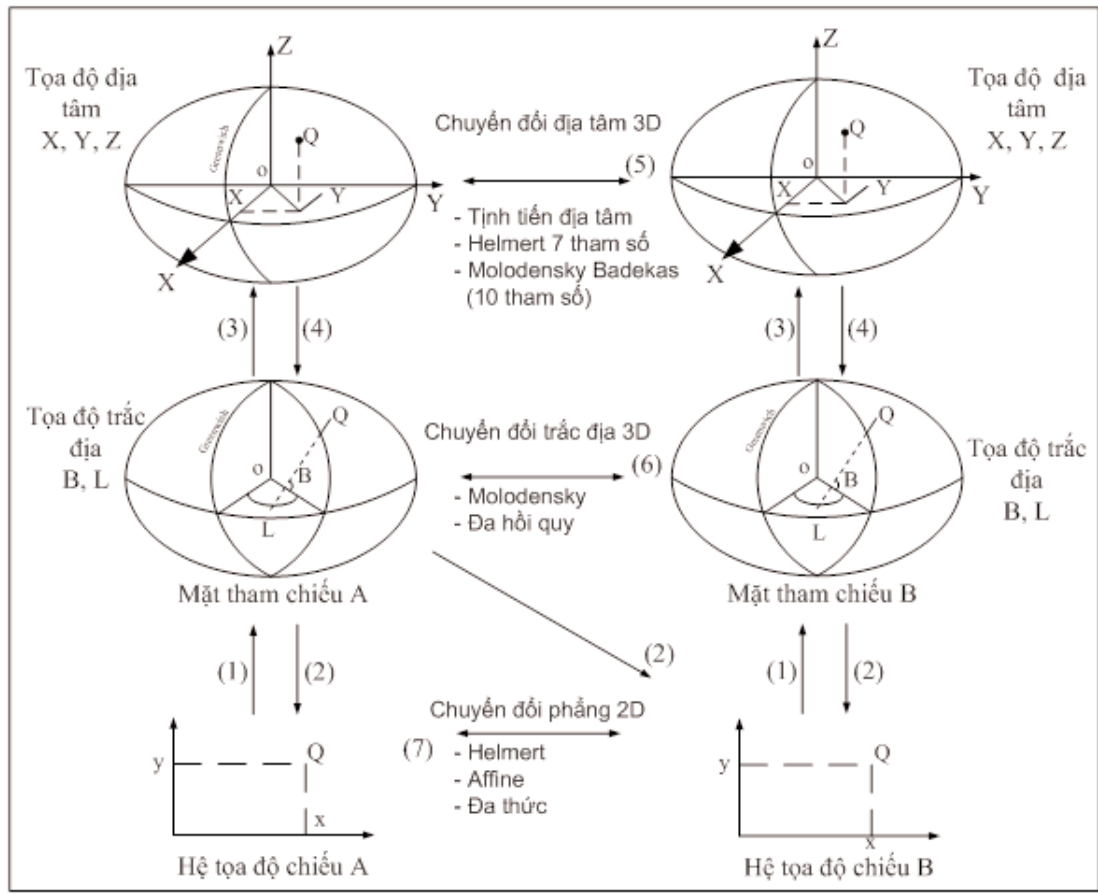

Hình 2: Các hệ thống tọa độ và các phương pháp chuyển đổi tọa độ qua lại

Bước 2: Chuyển đổi tọa độ địa tâm XYZ ở hệ WGS84 sang tọa độ địa tâm XYZ ở hệ VN-2000. Sử dụng công thức Bursa-Worlf theo [2] như sau:

$$
\begin{aligned}
& X_{V N-2000}=\Delta x+k \cdot(X+\Omega z . Y-\Omega y \cdot Z) \\
& Y_{V N-2000}=\Delta y+k \cdot(-\Omega z \cdot X+Y+\Omega x . Z) \\
& Z_{V N-2000}=\Delta z+k .(\Omega y . X-\Omega x . Y+Z)
\end{aligned}
$$

Bước 3: Chuyển đổi tọa độ địa tâm XYZ ở hệ VN-2000 sang tọa độ trắc địa $\mathrm{B}, \mathrm{L}$ ở hệ VN-2000, sử dụng công thức tính chuyển theo [3] như sau:

$$
\begin{aligned}
& \mathrm{B}=\operatorname{atan}\left[\left(\mathrm{Z}+\mathrm{e}^{2} \mathrm{~N} \sin (\mathrm{B})\right) /\left(\mathrm{X}^{2}+\mathrm{Y}^{2}\right)^{0.5}\right] \text { (tính lặp) } \\
& \mathrm{L}=\operatorname{atan}(\mathrm{Y} / \mathrm{X})
\end{aligned}
$$

Bước 4: Chuyển đổi tọa độ trắc địa $\mathrm{B}, \mathrm{L}$ ở hệ VN-2000 sang tọa độ phẳng $\mathrm{x}, \mathrm{y}$ ở hệ VN2000, sử dụng công thức tính chuyển theo [1] như sau:

$$
\begin{aligned}
\mathrm{X}=\quad & \left(\mathrm{X}+\frac{\mathrm{N} \cdot \sin \mathrm{B} \cdot \cos \mathrm{B}}{2} \mathrm{l}^{2}+\frac{5-\mathrm{t}+9 \cdot \eta^{2}+4 \cdot \eta^{4}}{24} \cdot \mathrm{N} \cdot \sin \mathrm{B} \cdot \cos ^{3} \mathrm{~B} \cdot \mathrm{l}^{4}+\right. \\
& \left.\frac{\left(61-58 \cdot \mathrm{t}+\mathrm{t}^{2}+270 \cdot \eta^{2}-300 \cdot t \cdot \eta^{2}\right.}{720} \cdot \mathrm{N} \cdot \sin \mathrm{B} \cdot \cos ^{5} \mathrm{~B} \cdot \mathrm{l}^{6}\right) \cdot \mathrm{k} \\
\mathrm{y}= & \left(N \cdot \cos B \cdot l+\frac{1-t+\eta^{2}}{6} \cdot N \cdot \cos ^{3} \mathrm{~B} \cdot l^{3}+\right. \\
& \frac{5-18 t+\mathrm{t}^{2}+14 \cdot \eta^{2}-58 \cdot t \eta^{2}+13 \cdot \eta^{4}-64 \cdot t \eta^{4}}{120} \cdot N \cdot \cos ^{5} \mathrm{~B} \cdot l^{2}+ \\
& \left.\frac{61-479 \cdot t+179 \cdot t^{2}-t^{3}}{5040} \cdot N \cdot \cos ^{7} \mathrm{~B} \cdot l^{7}\right) \cdot k+500000
\end{aligned}
$$




\section{Chuyển đổi tọa độ phẳng $\mathrm{x}, \mathrm{y}$ ở VN-2000 sang tọa độ trắc địa $\mathrm{B}, \mathrm{L}$ ở WGS84}

Bài toán chuyển đổi tọa độ này được thực hiện theo trình tự ngược lại với bài toán chuyển đổi tọa độ trắc địa $\mathrm{B}, \mathrm{L}$ ở WGS84 sang tọa độ phẳng $\mathrm{x}, \mathrm{y}$ ở VN-2000 đã trình bày ở mục 3. Quá trình tính chuyển tọa độ bao gồm 4 bước:

Bước 1: tính chuyển từ tọa độ phẳng $\mathrm{x}, \mathrm{y}$ ở hệ $\mathrm{VN}-2000$ sang tọa độ trắc địa $\mathrm{B}, \mathrm{L}$ ở $\mathrm{VN}$ 2000, sử dụng công thức tính chuyển theo [1] như sau:

$$
\begin{gathered}
\mathrm{L}=L_{0}+\frac{y}{N_{0} \cdot \cos B_{0}}-\frac{1}{6} \cdot\left(1+2 t_{0}+N_{0} \cdot \eta_{0}{ }^{2}\right) \cdot\left(\frac{y}{N_{0}}\right)^{3}+\frac{1}{120} \cdot\left(5+28 \cdot t_{0}+24 \cdot t_{0}^{2}+6 \cdot \eta_{0}{ }^{2}+\right. \\
\left.\quad 8 \cdot \eta_{0}^{2} t_{0}\right) \cdot\left(\frac{y}{N_{0}}\right)^{5}-\frac{1}{5040} \cdot\left(61+662 \cdot t_{0}+1320 \cdot t_{0}{ }^{2}+720 \cdot t_{0}{ }^{3}\right) \cdot\left(\frac{y}{N_{0}}\right)^{7} \\
\mathrm{~B}=B_{0}-\frac{1}{2} \cdot\left(1+\eta_{0}{ }^{2}\right) \cdot \operatorname{tg} B_{0}\left(\frac{y}{N_{0}}\right)^{2}+\frac{\left(1+\eta_{0}{ }^{2}\right)}{24} \cdot\left(5+3 \cdot t_{0}+\eta_{0}{ }^{2}-9 \cdot \eta_{0}{ }^{2} t_{0}\right) \cdot \operatorname{tg} B_{0}\left(\frac{y}{N_{0}}\right)^{4} \\
-\frac{\left(1+\eta_{0}^{2}\right)}{720} \cdot\left(61+90 \cdot t_{0}+45 \cdot t_{0}{ }^{2}+46 \eta_{0}{ }^{2}-720 \cdot \eta_{0}{ }^{2} \cdot t_{0}{ }^{2}\right) \cdot \operatorname{tg} B_{0} \cdot\left(\frac{y}{N_{0}}\right)^{6} \\
\quad+\frac{\left(1+\eta_{0}{ }^{2}\right)}{40320} \cdot\left(1385+3633 \cdot t_{0}+4095 \cdot t_{0}{ }^{2}+1575 t_{0}{ }^{2}\right) \cdot \operatorname{tg} B_{0} \cdot\left(\frac{y}{N_{0}}\right)^{8}
\end{gathered}
$$

Bước 2: Chuyển đổi tọa độ trắc địa $\mathrm{B}, \mathrm{L}$ ở hệ VN-2000 sang tọa độ địa tâm XYZ ở hệ VN-2000, sử dụng công thức tính chuyển theo [3] như sau:

$$
\begin{aligned}
& X=(N+H) \cos B \cos L \\
& Y=(N+H) \cos B \sin L \\
& Z=\left\{N\left(1-e^{2}\right)+H\right\} \sin B
\end{aligned}
$$

Bước 3: Chuyển đổi tọa độ địa tâm XYZ ở hệ VN-2000 sang tọa độ địa tâm XYZ ở hệ WGS84, sử dụng công thức tính chuyển theo [2] như đã trình bày ở bước 1 mục 3 nhưng điều chỉnh 7 tham số chuyển đổi cho phù hợp.

Bước 4: Tính chuyển từ tọa độ địa tâm XYZ ở WGS84 sang tọa độ trắc địa $\mathrm{B}, \mathrm{L}$ ở WGS84 theo nguyên tắc tính toán được thực hiện giống như bước 3 trong mục 3 .

\section{Xây dựng công cụ chuyển đổi tọa độ online và đánh giá khả năng tính toán chuyển đổi của công cụ}

Dựa vào các bước tính toán cụ thể đã triển khai bên trên từ khung lý thuyết của Knippers, R.A and Hendrikse J[4], một chương trình tính chuyển tọa độ đã được xây dựng dựa trên ngôn ngữ lập trình hướng đối tượng Python. Chương trình tính chuyển tọa độ này được lưu ở server. Phía client, một giao diện chuyển đổi tọa độ online được xây dựng và lưu trong một file .html giống như hình 3:

Để tính chuyển tọa độ từ WGS84 sang VN-2000, người nhập sẽ nhập vào các giá trị kinh độ (lon), vĩ độ (lat), độ rộng múi chiếu (zone), và kinh tuyến trung ương (Kt), tiếp theo sẽ nhấn nút $\gg$ để yêu cầu thực thi chuyển đổi tọa độ tại server, đoạn mã được sử dụng trong file .html để gọi thực thi hàm python WGS84_TO_VN2000.py ở server có cấu trúc như sau:

\section{\$.ajax(}




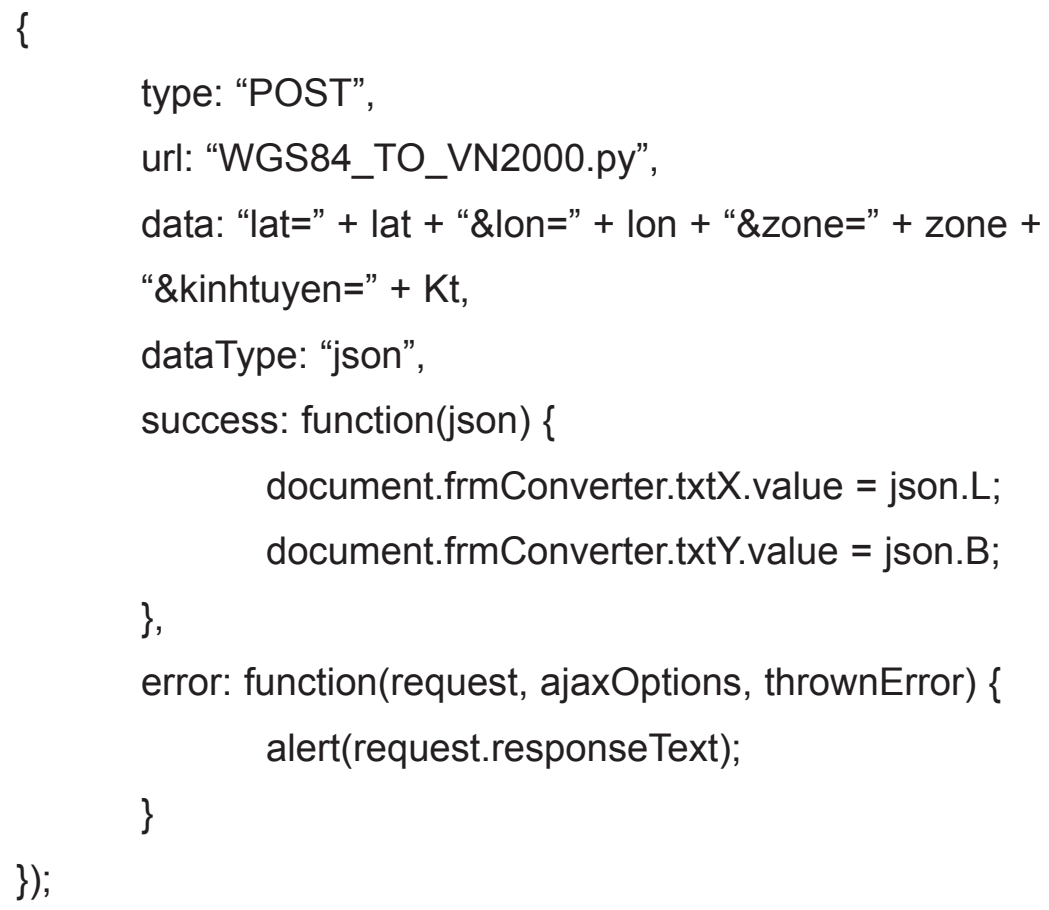

Nếu các thông số nhập vào hợp lệ, chương trình sẽ tính chuyển tọa độ và gán giá trị trả về vào các ô x (Đông), y (Bắc) trên giao diện hình 3 .

Để tính chuyển tọa độ từ VN-2000 sang WGS84, người nhập sẽ nhập vào các giá trị $x$ (Đông), y (Bắc), độ rộng múi chiếu (zone), và kinh tuyến trung ương (Kt), tiếp theo sẽ nhấn nút $\gg$ để yêu cầu thực thi chuyển đổi tọa độ tại server, đoạn mã được sử dụng trong file html để gọi thực thi ở server tương tự như mô tả ở trên, chỉ khác ở tên chương trình

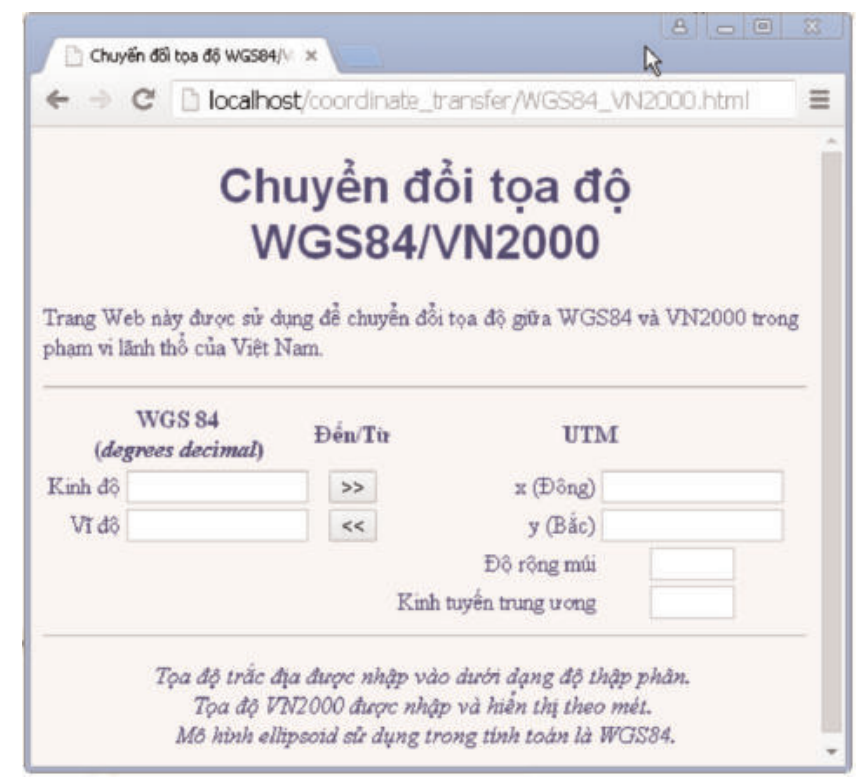

Hình 3: Giao diện của chương trình chuyển đổi tọa độ online 
python dùng tính chuyển tọa độ là VN2000_TO_WGS84.py.

Kết quả tính toán từ chương trình chuyển đổi tọa độ viết trên nền ngôn ngữ Python sau đó sẽ được so sánh với kết quả thực hiện chuyển đổi sử dụng Proj4js [5].Proj4js là một thư viện mã nguồn mở JavaScript dùng chuyển đổi tọa độ từ một hệ thống tọa độ này sang một hệ thống tọa độ khác, bao gồm cả chuyển đổi datum. Proj4js hiện đang được sử dụng rất phổ biến, đặc biệt trong lĩnh vực WebGIS. Điểm cần lưu ý là khi dùng Proj4js, người sử dụng không biết tường tận cơ sở lý thuyết của ứng dụng chuyển đổi này. Đây có thể là một mối bận tâm cho các nhà nghiên cứu phát triển. Nếu công cụ chuyển đổi vừa phát triển trên một cơ sở lý thuyết được diễn giải rõ ràng, lại cho kết quả tương ứng tốt với Proj4js, sẽ giải quyết được mối bận tâm nói trên.

Trường hợp 1: Tính chuyển tọa độ từ $B, L$ ở hệ WGS84 sang x,y ở hệ VN-2000.

Để xác định xem có sự khác biệt nào giữa tính toán như đã mô tả theo cơ sở lý thuyết triển khai bên trên và tính toán được thực hiện trong Proj4js, 100 điểm mẫu được tạo ngẫu nhiên nằm trong ranh giới Việt Nam có tọa độ $\mathrm{B}, \mathrm{L}$ ở hệ WGS84. Tiến hành chuyển đổi tọa độ các điểm này sang $x, y$ ở VN-2000 sử dụng múi chiếu $3^{\circ}$, kinh tuyến trung ương $105.75^{\circ}$ bằng hai cách: i) tính trực tiếp theo trình tự và công thức mô tả ở trên bằng Python và ii) sử dụng thư viện Proj4js. Bảng 1 minh họa chỉ một vài kết quả tính toán từ 2 phương pháp:

Bảng 1

\begin{tabular}{|c|c|c|c|c|c|}
\hline \multicolumn{2}{|c|}{ WGS84 } & \multicolumn{4}{c|}{ VN-2000 } \\
\hline L & B & X $_{\text {Python }}$ & Y $_{\text {Python }}$ & X $_{\text {Proj4js }}$ & Y $_{\text {Proj4js }}$ \\
\hline 103.355375 & 20.906553 & 250651.0551 & 2314467.2727 & 250651.0550 & 2314467.2655 \\
\hline 104.713263 & 19.748081 & 391147.9304 & 2184701.4213 & 391147.9304 & 2184701.4143 \\
\hline 104.805735 & 20.272345 & 401167.5706 & 2242683.2319 & 401167.5706 & 2242683.2248 \\
\hline 105.820713 & 10.046239 & 507555.4828 & 1110971.0561 & 507555.4829 & 1110971.0524 \\
\hline 102.808621 & 22.108839 & 196238.9809 & 2448650.8155 & 196238.9808 & 2448650.8079 \\
\hline
\end{tabular}

Kết quả thực hiện trên 100 điểm cho thấy có sự chênh lệch $D x$ và Dy giữa 2 cách tính như sau:

Min $(D x)=-0.1 \mathrm{~mm}$; $\operatorname{Max}(D x): 0.1 \mathrm{~mm}$; Độ lệch chuẩn: $0.05143199 \mathrm{~mm}$

Min $(D y)$ = 3.6mm; Max (Dy): 7.8 mm; Độ lệch chuẩn: 1.45899 mm

Mặc dù có sự khác biệt, tuy nhiên khác biệt là rất nhỏ, nên kết quả tính toán cho thấy có sự phù hợp giữa công cụ mới, xây dựng trên nền Python và công cụ thực hiện dựa trên thư viện Proj4js.

Trường hợp 2: Tính chuyển tọa độ từ $x, y$ ở hệ VN-2000 sang $B, L$ ở hệ WGS84.

Tương tự, để xác định xem có sự khác biệt nào giữa tính toán như đã mô tả theo cơ sở lý thuyết triển khai bên trên và tính toán được thực hiện trong Proj4js, 100 điểm mẫu được tạo ngẫu nhiên nằm trong ranh giới Việt Nam có tọa độ $x$, y ở hệ VN-2000 thuộc múi chiếu $3^{\circ}$, kinh tuyến trung ương $105.75^{\circ}$. Tiến hành chuyển đổi tọa độ các điểm này sang $\mathrm{B}, \mathrm{L}$ ở WGS84 sử dụng bằng hai cách: i) tính trực tiếp theo trình tự và công thức mô tả ở trên bằng Python và ii) sử dụng thư viện Proj4js. Bảng 2 minh họa chỉ một vài kết quả tính toán từ 2 phương pháp: 
Bảng 2

\begin{tabular}{|c|c|c|c|c|c|}
\hline \multicolumn{2}{|c|}{ VN-2000 } & \multicolumn{4}{c|}{ WGS84 } \\
\hline $\mathbf{x}$ & $\mathbf{y}$ & L $_{\text {Python }}$ & B $_{\text {Python }}$ & L $_{\text {Proj4js }}$ & B $_{\text {Proj4js }}$ \\
\hline 407610.6775 & 2407967.507 & 104.8585800169 & 21.765491520184 & 104.858580017156 & 21.765491503143 \\
\hline 318122.4013 & 2388280.721 & 103.9957031053 & 21.580835097631 & 103.995703105773 & 21.580835080808 \\
\hline 401167.5698 & 2242683.232 & 104.8057346005 & 20.272344961922 & 104.805734600724 & 20.272344947281 \\
\hline 698759.6015 & 1418434.772 & 107.5825348579 & 12.819648963716 & 107.582534857757 & 12.819648960566 \\
\hline 601835.209 & 957877.3595 & 106.6771139839 & 8.660817922206 & 106.677113983870 & 8.660817922964 \\
\hline
\end{tabular}

Kết quả thực hiện trên 100 điểm cho thấy có sự chênh lệch $D L$ và $D B$ giữa 2 cách tính như sau:

Min (Dx) = -0.000003 giây; Max (Dx): 0.000011 giây; Độ lệch chuẩn: 2.67945E-06 giây

Min (Dy) = -0.000008 giây; Max (Dy): 0.000069; Độ lệch chuẩn: 2.61214E-05 giây

Như vậy, trong tính toán chuyển đổi tọa độ từ $x, y$ ở hệ VN-2000 sang B, L ở hệ WGS84 sự khác biệt cũng là rất nhỏ giữa kết quả thực hiện của hai công cụ, một công cụ mới xây dựng theo cơ sở lý thuyết triển khai bên trên, và công cụ được thực hiện dựa trên thư viện Proj4js.

\section{Kết luận}

Hệ thống tọa độ quy định được sử dụng ở Việt Nam là hệ quy chiếu và hệ toạ độ quốc gia VN-2000 với mặt tham chiếu là ellipsoid quốc tế WGS84. Tuy nhiên, trong thực tế đo đạc, thu thập dữ liệu vị trí thông qua các thiết bị định vị GPS, tọa độ theo hệ tọa độ quốc tế WGS84 được ghi nhận, trước khi được các phần mềm chuyên dụng tính chuyển về hệ tọa độ VN-2000. Thay vì sử dụng phần mềm chuyển đổi tọa độ chuyên dụng, một hệ thống chuyển đổi tọa độ online được đề xuất sử dụng. Hệ thống được xây dựng theo mô hình client-server. Dựa trên cơ sở chung về lý thuyết chuyển đổi tọa độ, trình tự tính chuyển tọa độ qua lại giữa hệ tọa độ VN-2000 và hệ WGS84 được xác lập. Chương trình tính chuyển tọa độ online phía server được viết dựa trên ngôn ngữ Python và giao diện chuyển đổi tọa độ phía client được viết sử dụng Javascript. Để kiểm chứng khả năng của công cụ chuyển đổi tọa độ online vừa xây dựng, 100 điểm phân bố ngẫu nhiên trên phạm vi Việt Nam được tạo ra, và kết quả tính chuyển trên các điểm này sử dụng công cụ vừa xây dựng được so sánh với kết quả nhận được khi sử dụng thư viện mã nguồn mở Javascript nổi tiếng Proj4js. Thực nghiệm cho thấy công cụ vừa phát triển cho kết quả chuyển đổi rất sát với kết quả nhận được từ Proj4js. $O$ 


\section{Tài liệu tham khảo}

[1]. Hà Minh Hòa. Phương pháp chuyển đổi tọa độ giữa các hệ tọa độ. Nhà xuất bản Khoa học và Kỹ thuật, 2013.

[2]. Hướng dẫn 1123/ĐĐBĐ-CNTD của Cục Đo đạc Bản đồ. Hướng dẫn sử dụng các tham số tính chuyển từ hệ tọa độ quốc tế WGS84 sang Hệ Tọa Độ Quốc Gia VN-2000 và ngược lại.

[3]. IOGP Publication 373, GeomaticsGuidance Note Number 7, part 2, April 2015, International Association of oil and gas production.

[4]. Knippers, R.A and Hendrikse J.,Coordinate transformations. Kartografisch Tijdschrift, KernKatern 2000-3, 2001.

[5]. Proj4js, http://proj4js.org/

[6]. Quyết định số 83/2000/QĐ-TTg. Quyết định của Thủ tường Chính phủ về sử dụng hệ quy chiếu và hệ tọa độ quốc gia Việt Nam (VN-2000). O

\section{Summary}

\section{An Online coordinate converter to convert coordinates between VN-2000 and WGS84}

Assoc. Prof. Dr. Tran Trong Duc

Ho Chi Minh city University of Technology, VNU - HCM

As stipulatedby the 83/2000/QĐ-TTg Decision, map coordinates must be stored and displayed following the national coordinate system VN-2000. However, in practice, specialy in Global Positioning System field, coordinates are first recorded in WGS84 system and therefore later on have to be converted to $\mathrm{VN}-2000$. The conversion of coordinates back and forth can be performed by a number of specialized commercial softwares. However, instead of using specialized commercial softwares, this paper develops an online coordinate transformation system based on client/server model. The newly developed converter enables user with web browser, like firefox, to perform coordinate transfromation between the two coordinate systems without using specialized commercial softwares. To validate the capability of the proposed converter, an experiment is carried out in which coordinates of 100 random data points within Viet Nam boundary are transformed back and forth between the two coordinate systems. The results received from the proposed online coordinate converter are closed to the results received using open source javascript library Proj4js. $O$ 